\title{
Antioxidant vitamins supplementation and arterial stiffness: A systematic review and meta-analysis of randomised controlled trials
}

\author{
Ammar W. Ashor ${ }^{1,2}$, Mario Siervo ${ }^{1}$, Clio Oggioni ${ }^{1}$, Jose Lara ${ }^{1}$ and John C. Mathers ${ }^{1}$ \\ ${ }^{1}$ Human Nutrition Research Centre, Institute for Ageing and Health, Newcastle University, Campus for Ageing and \\ Vitality, Newcastle on Tyne, NE4 5PL, UK and ${ }^{2}$ College of Medicine, University of Al-Mustansiriyah, Baghdad, Iraq
}

Inflammation and oxidative stress contribute significantly to arterial stiffness ${ }^{(1)}$. Several studies have tested the effects of supplementation with antioxidant vitamins on arterial stiffness (a precursor of cardiovascular diseases) but the results have been contradictory ${ }^{(2,3)}$. Our objective was to conduct a systematic review and meta-analysis investigating the effect of antioxidant vitamins on arterial stiffness. The second objective was to determine whether the effects on arterial stiffness vary according to dose of antioxidant vitamins, duration of intervention, and health or nutritional status of the included participants.

We searched 3 databases (Medline, Embase and Scopus) for articles with the following criteria: 1) randomized controlled trials comparing antioxidant vitamins (vitamins C, E, A, beta-carotene) with either placebo or no active control; 2) adult participants $\geqslant 18$ years; 3) antioxidant vitamins administered alone or in combination; 4) changes in arterial stiffness or arterial compliance. Data were pooled as standardized mean difference (SMD) and analysed using a fixed effects model.

Pooled analysis (20 studies, 1,909 participants) showed that antioxidant vitamins supplementation reduced arterial stiffness significantly (SMD: $-0 \cdot 17,95 \% \mathrm{CI}$ : $-0 \cdot 26,-0 \cdot 08, P=0 \cdot 0001$ ). This effect was significant in both experimental and primary prevention studies but not in studies including participants with diseases (SMD: $-0 \cdot 19,95 \% \mathrm{CI}:-0 \cdot 40,-0 \cdot 02, P=0 \cdot 081$ ). Vitamins supplementation improved arterial stiffness irrespective of age group and duration of intervention. Antioxidant vitamins were more effective in participants with low baseline plasma concentrations of vitamin $\mathrm{C}$ and $\mathrm{E}$.

In conclusion, supplementation with antioxidant vitamins has a small, protective effect on arterial stiffness. The effect may be augmented in those with lower baseline plasma vitamin $\mathrm{E}$ and $\mathrm{C}$ concentrations.

1. Shirwany NA, Zou MH (2010). Acta pharmacologica Sinica 31, 1267-1276.

2. Plantinga Y, Ghiadoni L, Magagna A et al. (2007). American Journal of Hypertension 20, 392-397.

3. Zureik M, Galan P, Bertrais S et al. (2004). Arteriosclerosis, Thrombosis \& Vascular Biology 24, 1485-1491. 